\title{
UNSTEADY WAVES ON AN OPEN TWO LAYER FLUID
}

\author{
P. W. SHARP ${ }^{1}$
}

(Received 19 August 1982; revised 16 August 1983)

\begin{abstract}
The evolution of small amplitude waves on an open two layer fluid is investigated. The spatially periodic surface and interface displacements are represented as Fourier series with time dependent coefficients, for which evolution equations with all significant quadratic interactions included, are derived. Solutions to these equations are found analytically for a small number of harmonics, and numerically for a larger number of harmonics. Two numerical solutions are given to illustrate the evolution properties.
\end{abstract}

\section{Introduction}

The classical theory of gravity wave propagation based on a linearised form of the equation of motion has wave solutions which propagate independently of one another, with no energy transfer between waves. When the nonlinear interactions are included Phillips [8] has shown that if the interactions occur over a sufficiently long time, and if a resonance condition (to be stated later) is satisfied, then there will be a significant transfer of energy between the waves. Phillips found for gravity waves on a deep single layer fluid that the interaction rate was of $O\left(\varepsilon^{2}\right)$ where $\varepsilon \ll 1$ is a measure of the wave amplitude. Thus for a complete description of the evolution of the wave field over a time interval of $O\left(\varepsilon^{-2}\right)$ the linear theory must be extended to include the nonlinear terms. Following Phillips, Bretherton [3] introduced the idea of near resonance where the resonance condition is satisfied to $O(\varepsilon)$.

When the fluid, because of the presence of a thermocline or a layer of freshwater upon saltwater must be regarded as a two layer fluid the wave evolution is more complicated. It has long been known (see for example Lamb [7])

\footnotetext{
${ }^{1}$ Department of Mathematics, University of Canterbury, Christchurch 1, New Zealand. Present address: Department of Computer Science, University of Toronto, Toronto, Canada M5S 1A4.

(c) Copyright Australian Mathematical Society 1984, Serial-fee code 0334-2700/84
} 
that two types of wave modes are possible. One is a fast surface mode and the other is a slow interface mode. Ball [1] has shown that when both types of modes are present with the interface waves long compared with the surface waves, resonance is possible between two surface waves and one interface wave. The resonance condition is then, for unidirectional propagation

$$
\omega_{k+l}-\omega_{l}-\omega_{k}=0, \quad l \gg k,
$$

where $\omega_{k+l}, \omega_{l}$ are the frequencies of the two surface modes with wavenumbers $k+l, l$, and $\omega_{k}$ is the frequency of the interface mode with wavenumber $k$.

The perturbation scheme used by Phillips [8] shows that this type of interaction occurs at an $O(\varepsilon)$ rate. Thus for gravity waves on deep water with both surface-surface and surface-interface wave interactions present the latter interaction dominates the wave field evolution up to a time of $O\left(\varepsilon^{-1}\right)$.

The evolution of gravity waves on a two layer fluid has been studied theoretically by several authors. Ball [1] for shallow water, and Hashizume and Ikeda [6] for arbitrary depths have found analytic solutions for two surface waves and one interface wave satisfying equation (1.1). Watson et al [13] considered the interaction between linear surface and interface wave fields, while Rizk and Ko [10] studied the interaction between surface Stokes waves and large scale internal waves.

The approach used here has both the interface and surface displacement as unknown functions, resolved into as many wave modes as are required (to be defined more precisely in Section 5). To simplify the algebra we have taken the wave propagation to be unidirectional and the lower layer to be of depth far greater than any wavelengths present. A limit is placed on the wave slopes to exclude large amplitude waves.

The waves are represented as spatially periodic Fourier series with coefficients which vary slowly with time (see Bretherton [3] for a precise definition of slowly varying in time). Evolution equations with all the significant quadratic interactions included are derived for the rates of change of the Fourier coefficients. The cubic and higher order interactions are omitted because as stated above they occur on a longer time scale than the quadratic interactions.

Several analytic results are given but in general numerical methods must be used to solve the evolution equations. Two contrasting numerical solutions are given and these are used to illustrate general properties of all the solutions found, as well as properties that are unique to different sets of solutions.

\section{Governing equations}

The two layer fluid consists of an upper layer of density $\rho_{2}$, mean depth $h$ and a lower layer of density $\rho_{1}$ and infinite depth with the difference $\rho_{1}-\rho_{2}$ being small compared with $\rho_{1}$ and $\rho_{2}$. The fundamental wavelength is $2 \pi L$ and $a_{1}, a_{2}$ 
are a measure of the amplitude of the interface and surface waves respectively. The horizontal coordinate $x$ and the vertical coordinate $y$ (positive upwards) are both in units of $h$ with the origin on the mean interfacial level. The interface and surface displacements are $\eta_{1}$ and $\eta_{2}$ respectively, both in units of $a_{1}$ and the corresponding velocity potentials are $\phi_{1}$ and $\phi_{2}$ in units of $(g h)^{1 / 2} a_{1}$, where $g$ is gravity. The time $t$ is in units of $(h / g)^{1 / 2}$ and the principal parameter is $\varepsilon=a_{1} / h \ll 1$. The other parameters are $\mu=h / L, \varepsilon^{\prime}=a_{2} / h \ll 1$ and $\rho=\rho_{1} / \rho_{2}$.

The governing equations for this inviscid, incompresible and irrotational flow are:

(i) Laplace's equation for the velocity potentials $\phi_{1}, \phi_{2}$;

(ii) the bottom boundary condition;

(iii) the interface kinematic and dynamic boundary conditions;

(iv) the surface kinematic and dynamic boundary conditions.

When the surface and interface boundary conditions are expanded in $\varepsilon$ about $y=1$ and 0 respectively the governing equations become

$$
\begin{gathered}
\phi_{2 x x}+\phi_{2 y y}=0, \quad 0<y<1, \\
\phi_{1 x x}+\phi_{1 y y}=0, \quad-\infty<y<0, \\
\phi_{1 y} \rightarrow 0 \text { as } y \rightarrow-\infty, \\
\phi_{1 y}-\eta_{1 t}=\varepsilon\left(\eta_{1} \phi_{1 x}\right)_{x}+O\left(\varepsilon^{2}\right) \quad \text { on } y=0, \\
\phi_{2 y}-\eta_{1 t}=\varepsilon\left(\eta_{1} \phi_{2 x}\right)_{x}+O\left(\varepsilon^{2}\right) \quad \text { on } y=0, \\
\rho \phi_{1 t}-\phi_{2 t}+(\rho-1) \eta_{1}=\varepsilon \eta_{1} \phi_{2 y t}-\varepsilon \rho \eta_{1} \phi_{1 y t}+\frac{1}{2} \varepsilon\left(\phi_{2 x}^{2}+\phi_{2 y}^{2}\right) \\
-\frac{1}{2} \varepsilon \rho\left(\phi_{1 x}^{2}+\phi_{1 y}^{2}\right)+O\left(\varepsilon^{2}\right) \quad \text { on } y=0, \\
\phi_{2 y}-\eta_{2 t}=\varepsilon\left(\eta_{2} \phi_{2 x}\right)_{x}+O\left(\varepsilon^{2}\right) \quad \text { on } y=1, \\
\phi_{2 t}+\eta_{2}=-\varepsilon \eta_{2} \phi_{2 y t}-\frac{1}{2} \varepsilon\left(\phi_{2 x}^{2}+\phi_{2 y}^{2}\right)+O\left(\varepsilon^{2}\right) \quad \text { on } y=1 .
\end{gathered}
$$

The details of the derivation can be found in Sharp ([12], pages 7-8).

Spatially periodic solutions of the form

$$
\begin{aligned}
& \eta_{1}=\frac{1}{2} \sum_{k=1}^{\infty} A_{1 k}(t) \exp i\left(k \mu x-\omega_{k} t\right)+*, \\
& \eta_{2}=\frac{1}{2} \sum_{k=1}^{\infty} A_{2 k}(t) \exp i\left(k \mu x-\omega_{k} t\right)+*, \\
& \phi_{1}=\frac{1}{2} \sum_{k=1}^{\infty} B_{1 k}(t) e^{k \mu y} \exp i\left(k \mu x-\omega_{k} t\right)+*,
\end{aligned}
$$




$$
\phi_{2}=\frac{1}{2} \sum_{k=1}^{\infty}\left(B_{2 k}(t) \cosh k \mu y+C_{2 k}(t) \sinh k \mu y\right) \exp i\left(k \mu x-\omega_{k} t\right)+*
$$

are sought where * denotes the complex conjugate, $k$ is the wavenumber in units of $L^{-1}$ and $\omega_{k}$ is the frequency in units of $(g / h)^{1 / 2}$.

The linear solutions to equations (2.1) are found by substituting in the above Fourier series and neglecting the right hand sides. This gives

$$
\begin{aligned}
A_{1 k} & =\left(1-T_{k} k \mu / \omega_{k}^{2}\right) \cosh k \mu A_{2 k}=\left(i k \mu / \omega_{k}\right) B_{1 k} \\
& =\left(i k \mu / \omega_{k}\right) C_{2 k} \\
& =\left(i k \mu / \omega_{k}\right)\left(\omega_{k}^{2} / k \mu-T_{k}\right) /\left(1-T_{k} \omega_{k}^{2} / k \mu\right) B_{2 k}
\end{aligned}
$$

and the dispersion relation

$$
\left(\rho+T_{k}\right)\left(\omega_{k}^{2} / k \mu\right)^{2}-\rho\left(1+T_{k}\right) \omega_{k}^{2} / k \mu+(\rho-1) T_{k}=0
$$

where $T_{k}=$ Tanh $k \mu$.

The two roots of this equation are

$$
\omega_{A k}^{2} / k \mu=1
$$

and

$$
\omega_{B k}^{2} / k \mu=(\rho-1) T_{k} /\left(\rho+T_{k}\right) .
$$

The larger root $\omega_{A k}$ is the frequency of a free surface wave mode and is the same as for waves on deep water, indicating that these modes propagate independently of the properties of the interface. The smaller root $\omega_{B k}$ is the frequency of a free interface wave mode and depends on the reduced gravity $(\rho-1)$.

The evolution equations for $A_{1 k}$ and $A_{2 k}$ are now derived by evaluating the nonlinear terms in equations (2.1) and eliminating $B_{1 k}, B_{2 k}$ and $C_{2 k}$ to give two second order ordinary differential equations involving $A_{1 k}$ and $A_{2 k}$. One of these equations can be integrated twice and the other once to give the equations which form the basis of our paper. The details of this method of elimination and subsequent integrations are given in Bryant [5]. Only the important points will be presented here.

The method of eliminating $B_{1 k}, B_{2 k}$ and $C_{2 k}$ depends on whether they occur in the linear or nonlinear terms. If they occur in the linear terms they must be found by simultaneous solution. If they occur in the nonlinear terms then it is possible to substitute for them in terms of $A_{1 k}$ and $A_{2 k}$ from equations (2.3). With $\varepsilon$ nonzero the values of $B_{1 k}, B_{2 k}$ and $C_{2 k}$ differ from those in equations (2.3) by $O(\varepsilon)$. This difference, when included in the nonlinear terms, becomes of $O\left(\varepsilon^{2}\right)$ and hence is neglected. 
The resulting differential equations, after the integrations, become

$$
\begin{aligned}
A_{1 k}- & \left(1-T_{k} k \mu / \omega_{k}^{2}\right) \cosh k \mu A_{2 k} \\
= & -\frac{1}{2} \varepsilon \sum_{l=1}^{k-1} \frac{R_{D k,-l} A_{1 l} A_{1 k-l}}{\left(\omega_{l}+\omega_{k-l}\right)^{2}-\left(\omega_{A k} \omega_{B k} / \omega_{k}\right)^{2}} \exp \left(-i\left(\omega_{l}+\omega_{k-l}-\omega_{k}\right) t\right) \\
& -\varepsilon \sum_{l=1}^{\infty} \frac{R_{D k, l} A_{1 l}^{*} A_{1 k+l}}{\left(\omega_{k+l}-\omega_{l}\right)^{2}-\left(\omega_{A k} \omega_{B k} / \omega_{k}\right)^{2}} \exp \left(-i\left(\omega_{k+l}-\omega_{l}-\omega_{k}\right) t\right) \\
& +O\left(\varepsilon^{2}\right), \quad k=1,2, \ldots,
\end{aligned}
$$

and

$$
\begin{aligned}
& D\left\{A_{2 k}+(\rho-1)\left(1-T_{k} k \mu / \omega_{k}^{2}\right) \cosh k \mu A_{1 k}\right\} \\
& \quad=\frac{1}{2} i \varepsilon \sum_{l=1}^{k-1} \frac{R_{E k,-l} A_{1 l} A_{1 k-l}}{\left(\omega_{l}+\omega_{k-l}+\omega_{k}\right)} \exp \left(-i\left(\omega_{l}+\omega_{k-l}-\omega_{k}\right) t\right) \\
& \quad+i \varepsilon \sum_{l=1}^{\infty} \frac{R_{E k, l} A_{1 l}^{*} A_{1 k+l}}{\left(\omega_{k+l}-\omega_{l}+\omega_{k}\right)} \exp \left(-i\left(\omega_{k+l}-\omega_{l}-\omega_{k}\right) t\right)+O\left(\varepsilon^{2}\right), \\
& k=1,2, \ldots
\end{aligned}
$$

where $D=d / d t$ and the frequencies are extended to $k<0$ by defining $\omega_{k}=-\omega_{-k}$. The coefficients $R_{D k, l}$ and $R_{E k, l}$ are given in the Appendix.

On comparing the left hand sides of equations (2.4a) with the first equality in equations (2.3) we see that the right hand sides of equations (2.4a) give the quadratic corrections to the linear solutions. The right hand sides of equations (2.4a) contains factors of the form $\left(\omega_{1}+\omega_{k-1}-\omega_{A k} \omega_{B k} / \omega_{k}\right)^{-1}$ and $\left(\omega_{k+1}-\omega_{l}-\right.$ $\left.\omega_{A k} \omega_{B k} / \omega_{k}\right)^{-1}$. It will be shown in the next section that these are of $O(1)$ and hence the integration used above is valid. Finally while it is possible at this stage to obtain an expression for $d A_{1 k} / d t$ from equation (2.4a) and substitute into equation (2.4b) to obtain a differential equation for $A_{2 k}$, this is not done until the next section after the final form of the nonlinear terms is given.

\section{Evolution equations}

The final form of the nonlinear terms in equations (2.4a) and (2.4b) depends on which quadratic interactions are included. The possible interactions are outlined below and reasons given for including or excluding them.

A. Two interface wave modes interacting with a third interface wave. Equation (1.1) requires

$$
\left(\frac{(k+l) \mu T_{k+l}}{\rho+T_{k+l}}\right)^{1 / 2}-\left(\frac{l \mu T_{l}}{\rho+T_{l}}\right)^{1 / 2}-\left(\frac{k \mu T_{k}}{\rho+T_{k}}\right)^{1 / 2}=0 .
$$


In general this is impossible to satisfy but for long interface waves the left hand side of the above condition is of $O\left(\mu^{2}\right)$. The near resonance condition will then be satisfied when $\varepsilon / \mu^{2}=O(1)$. Because $\left(\omega_{B k+l}-\omega_{B l}-\omega_{A k}\right)$ is of $O(1)$ equation (2.4a) is valid. Therefore this interaction is included in order to consider long interface waves such that $\varepsilon / \mu^{2}=O(1)$.

B. Two surface wave modes interacting with an interface wave mode. Equation (1.1) requires

$$
((k+l) \mu)^{1 / 2}-(l \mu)^{1 / 2}-\left(\frac{(\rho-1) T_{k} k \mu}{\rho+T_{k}}\right)^{1 / 2}=0, \quad k+l, l \gg k .
$$

This can be satisfied by taking

$$
\mu=\frac{1}{2 k} \ln \{(\rho-1)(1+\beta) /[(\rho-1)-(\rho+1) \beta]\}
$$

where $\beta=(2 l / k)+1-2\left((l / k)^{2}+l / k\right)^{1 / 2}$.

Because $\left(\omega_{A k+1}-\omega_{A l}-\omega_{A k}\right)$ is of $O(1)$, equation (2.4a) is valid and this interaction is included.

C. An interface wave mode and a surface wave mode interacting with a surface wave mode. This is similar to case B above and is included.

Two surface wavemodes may interact with a third surface wave mode. Equation (1.1) would require $\omega_{A k+1}-\omega_{A l}-\omega_{A k}$ to be zero. As stated above this is of $O(1)$ and hence the interaction is excluded.

Finally an interface wave mode and a surface wave mode may interact with an interface wave mode or two interface wave modes may interact with a surface wave mode. These interactions are excluded as the smallness of the interface wavenumbers compared with the surface wavenumbers makes it impossible to satisfy the resonance or near resonance condition.

Benney [2] has shown that for short surface waves and long interface waves, the resonance condition (1.1) with $\varepsilon=0$ is equivalent to requiring the group speed of the surface waves to equal the phase speed of the interface waves, with the centre of the surface waveband at $k$. When $\varepsilon$ is nonzero this interpretation is not strictly true. However we will be considering solutions with $\varepsilon \ll 1$ and thus the centre of the waveband will be in a small neighbourhood of $k$. In this sense a frame of reference moving with the speed $d \omega_{A k} / d k \mu$ in the positive $x$ direction represents the 'best' frame in which to investigate the nonlinear interaction.

Therefore solutions of the form

and

$$
\eta_{1}=\frac{1}{2} \sum_{k=1}^{n} a_{1 k}(t) \exp i(k \mu(x-c t))+*
$$

are sought, where

$$
\eta_{2}=\xi(x, t) \exp i\left(K \mu x-\omega_{A K} t\right)+*
$$

$$
\xi(x, t)=\frac{1}{2} \sum_{k=l_{2}}^{u_{2}} a_{2 k}(t) \exp i((k-K) \mu(x-c t))
$$


and $c=d \omega_{A K} / d k \mu$. The $a_{1 k}$ and $a_{2 k}$ are complex and $\xi(x, t)$ is the envelope function. If the $a_{1 k}$ and $a_{2 k}$ are real and constant then the expression for $\eta_{2}$ is that of a wave group of central wavenumber $K$ and central frequency $\omega_{A K}$ propagating in the positive $x$ direction with a group speed $c$. The expression for $\eta_{1}$ is then that of a wave moving in phase with the surface envelope with phase speed $c$.

As stated in Section 1 the long interface wave modes are important. Therefore in equation (1.1) we take $k=1$ and choose $\mu$ for a given $K$ such that

$$
\frac{d \omega_{A K}}{d k \mu}=\frac{\omega_{B 1}}{\mu} \text {. }
$$

Expressing the complex amplitudes $A_{1 k}$ and $A_{2 k}$ from equations (2.2a) and (2.2b) in terms of $a_{1 k}$ and $a_{2 k}$ leads to the following evolution equations for $a_{1 k}$ and $a_{2 k}$;

$$
\begin{gathered}
\frac{d a_{1 k}}{d \tau}+\frac{i\left(\omega_{B k}-k \mu c\right)}{\varepsilon} a_{1 k}=\frac{1}{2} i \sum_{l=1}^{k-1} R_{A k,-l} a_{1 l} a_{1 k-l} \\
+i \sum_{l=1}^{n-k} R_{A k, l} a_{1 l}^{*} a_{1 l+k}+i \sum_{l=l_{2}}^{u_{2}-k} R_{B k, l} a_{2 l}^{*} a_{2 k+l}+O(\varepsilon), \\
\frac{d a_{2 k}}{d \tau}+\frac{i\left(\omega_{A k}-(k-K) \mu c-\omega_{A K}\right)}{\varepsilon} a_{2 k}=i \sum_{l=1}^{\min \left(n, k-l_{2}\right)} R_{C k,-l} a_{1 l} a_{2 k-l} \\
+i \sum_{l=1}^{\min \left(n, u_{2}-k\right)} R_{C k, l} a_{1 l}^{*} a_{2 k+l}+O(\varepsilon), \quad(3.2 \mathrm{a}) \\
l_{2} \leqslant k \leqslant u_{2}, \quad(3.2 \mathrm{~b})
\end{gathered}
$$

where the interaction coefficients $R_{A}, R_{B}, R_{C}$ are given in the Appendix and $\tau=\boldsymbol{\varepsilon} t$.

\section{Analytical results}

Defining $s_{l_{2}, u_{2}}^{n}$ to be the set of equations (3.2a) and (3.2b) with $\mu$ chosen such that the equation (1.1) is satisfied for some $k \in\left[l_{2}, u_{2}\right]$ the following analytical solutions are known.

For $s_{l_{2}, l_{2}+1}^{1}$, Hashizume and Ikeda [6] have given a full set of solutions. Expressing their solutions in our notation with the initial conditions $a_{11}=0$, $a_{2 l_{2}}=a_{2 l_{3}}=A\left(A\right.$ real, $\left.l_{3}=l_{2}+1\right)$ we obtain

$$
\begin{gathered}
\left|a_{11}(\tau)\right|=\beta^{1 / 2}|\operatorname{cn}(\omega \tau-T)|, \\
\left|a_{2 \prime_{2}}(\tau)\right|=A(1+\alpha)^{1 / 2} \operatorname{dn}(\omega \tau-T),
\end{gathered}
$$




$$
\begin{gathered}
\left|a_{2 l_{3}}(\tau)\right|=A|\operatorname{sn}(\omega \tau-T)|, \\
R_{B 1, l_{2}}\left|a_{2 l_{3}}\right|^{2}+R_{C l_{2,-1}\left|a_{11}\right|^{2}=R_{B 1, l_{2}} A^{2},} \\
R_{B 1, l_{2}}\left|a_{2 l_{2}}\right|^{2}-R_{C l_{2},}\left|a_{11}\right|^{2}=R_{B 1, l_{2}} A^{2}, \\
a_{11}^{*} a_{2 l_{2}}^{*} a_{2 l_{3}}+a_{11} a_{2 l_{2}} a_{2 l_{3}}^{*}=0,
\end{gathered}
$$

where $\mathrm{cn}, \mathrm{dn}, \mathrm{sn}$ are the Jacobi elliptic functions,

$$
\begin{aligned}
& \beta=R_{B 1, l_{2}} / R_{C l_{3},-1}, \quad \omega=\left(A^{2} R_{C l_{3},-1} R_{B 1, l_{2}}(1+\alpha)\right)^{1 / 2}, \\
& \alpha=R_{C l_{2}, 1} / R_{C l_{3},-1} \text { and } \quad T=\int_{0}^{\pi / 2} \frac{d \phi}{\sqrt{1-\frac{\alpha}{1+\alpha} \sin ^{2} \phi}}
\end{aligned}
$$

with $R_{B 1, l_{2}}, R_{C l_{2,1}}$ and $R_{C l_{3},-1}$ all greater than zero.

The essential features of this solution are the strict periodicity, the inverse dependence of the period on the initial amplitude, and the maximum value of the interface wave mode being $\beta A$ where $\beta<1$ in general.

When either the number of surface or interface wave modes was increased it was not possible to solve analytically the corresponding system of evolution equations. However for $s_{l_{2}, u_{2}}^{1}$ the following two independent complete integrals were found.

$$
\left|a_{11}\right|^{2}+\sum_{\substack{i=l_{2} \\ i \neq m}}^{u_{2}} \alpha_{i}\left|a_{2 i}\right|^{2}=C_{1}, \quad l_{2} \leqslant m \leqslant u_{2}
$$

and

$$
\sum_{i=I_{2}}^{u_{2}} \beta_{i}\left|a_{2 i}\right|^{2}=C_{2}
$$

where $\alpha_{i}, \beta_{i}$ are functions of the interaction coefficients and $C_{1}, C_{2}$ are constants determined by the initial conditions. The explicit form of $\alpha_{i}, \beta_{i}$ is not given because other complete integrals were used in subsequent calculations. Similarly for $s_{l_{2}, l_{2}+2}^{2}$ one complete integral of the above form involving all five wave modes was found. From these analytical results it can be seen that numerical methods are required to solve the evolution equations when an arbitrary number of wave modes are present. One such method is outlined in Section 5. However because of their nearness to resonance the evolution of the wave modes $a_{11}, a_{2 \ell_{2}}$ and $a_{2 l_{3}}$ as given in equation (4.1) dominates these numerical solutions. A way of measuring the degree of dominance is given in Section 5. 


\section{Numerical solutions}

The numerical method used to solve the evolution equations is the integrator of Shampine and Gordon ([11], pages 156-223). Briefly, their integrator is a variable order, variable stepsize, linear multistep method using an Adams-Bashforth predictor and an Adams-Moulton corrector. The integrator endeavours to keep the local error at each step below a value set by the user and at the same time using as large a stepsize as possible.

This is achieved by varying the order and stepsize. An estimate of the global error at the end of the integration can be found by resolving the equations with a more severe local error tolerance and noting the change in the solution. This method of estimating the global error is explained more fully in ([11], pages 122-125) and the results of numerical experiments on equations (3.2) using STEP are given in Sharp ([12], pages 43-45).

For a particular $\rho$ and $K, \mu$ was found such that $d \omega_{A K} / d k \mu=\omega_{B 1} / \mu$. Then for a given set of $\rho, K, \mu$ and $\varepsilon, s_{l_{2}, u_{2}}^{n}$ was solved numerically for a small number of wave modes. The number of modes was increased until the last mode added altered the previous solution by less than $10^{-2}$. This last mode was then excluded and the previous solution taken as the final solution. The initial conditions were

$$
\begin{aligned}
& a_{1 k}=0, \quad k=1, \ldots, n, \\
& a_{2 k}=0, \quad k=l_{2}, \ldots, K-1, K+2, \ldots, u_{2}, \\
& a_{2 K}=a_{2 K+1}=A
\end{aligned}
$$

where $A$ was chosen so that the time average of $\sum_{k \text { odd }}\left|a_{1 k}\right|$ was approximately 1 to be consistent with the definition of $a_{1}$. The parameter $\varepsilon$ was chosen so that the initial slope of $a_{2 k}$ was approximately 0.1 .

The complete integrals (4.1d) and (4.1e) were combined to give

$$
\left|a_{2 l_{3}}\right|^{2}+\left|a_{2 l_{2}}\right|^{2}+\left[\left(R_{C L_{3},-1}-R_{C l_{2}, 1}\right) / R_{B 1, l_{2}}\right]\left|a_{11}^{2}\right|=2 A^{2}
$$

For each numerical solution the time average of the left hand side of equation (5.2) was expressed as a fraction of $2 A^{2}$. This fraction was used as a measure of the dominance of the wave modes $a_{11}, a_{2 l_{2}}$ and $a_{2 / 3}$ over the remaining modes. Because $R_{\mathrm{Cl}_{3},-1} \simeq R_{\mathrm{Cl}_{2}, 1}$ equation (5.2) gives (see for example Phillips [9]) an estimate of the energy of $a_{2 l_{2}}$ and $a_{2 / 3}$, a result that is used later.

All the numerical solutions were found on a Prime 750 using double precision arithmetic with fourteen significant figures. 


\section{Numerical examples}

In this section two numerical solutions are given for two values of $\mu$. These two solutions are used to illustrate the general properties of all solutions found and also properties that apply only to subsets of our solutions with each subset characterized by a unique value of $\mu$.

The first example has $\rho=1.05, \varepsilon=.015, A=.8$ and $K=22$ giving $\mu=.319$. Eight interface modes and twenty-five surface modes with wavenumbers eleven to thirty-five were required to describe the solution fully as outlined in Section 5 . The interface displacement and surface envelope are given in Figures 1(a) and 1(b) for two different time intervals.

The first general principle as seen from the figures is that although the evolution is oscillatory it is not strictly periodic. The integration was continued until $\tau=400$ for this example and at the end of the integration the solution had
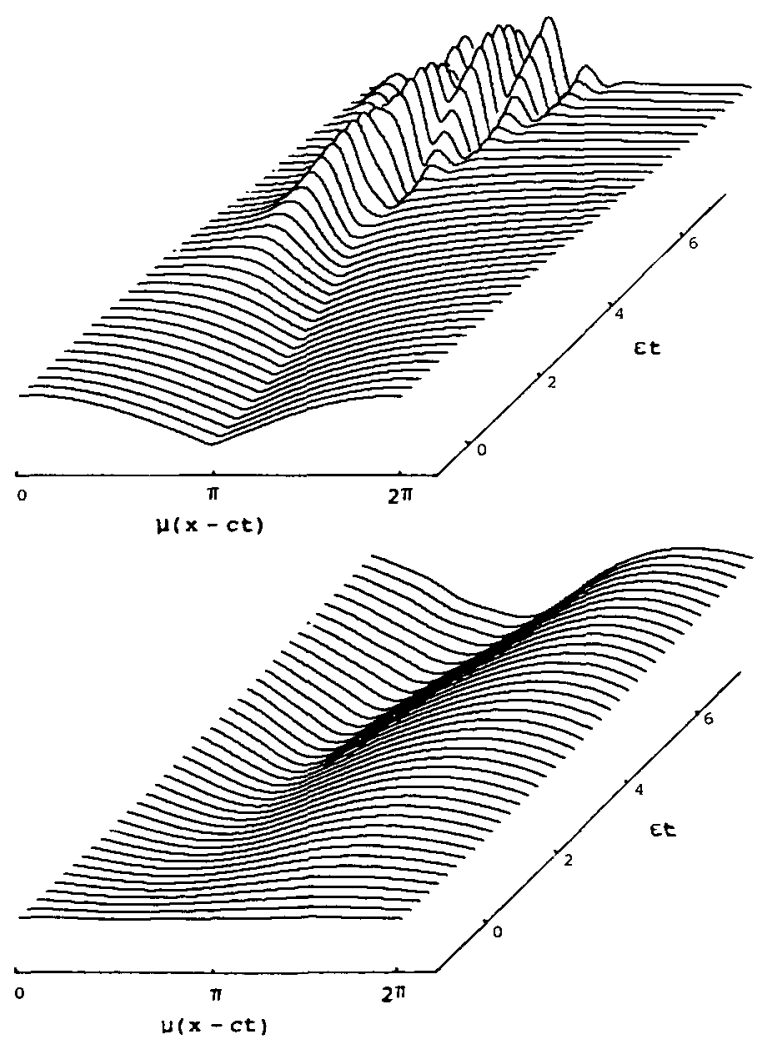

Figure 1(a). One wavelength of the surface envelope (upper graph) and interface displacement for $\tau=0-9$ with $\rho=1.05, \varepsilon=0.015, \mu=.319$ and $K=22$. The vertical magnification is 25 . 
not become steady or periodic. However because of the oscillatory evolution it was found possible to refer to a period of oscillation. This was taken to be that of $\left|a_{11}\right|$ and was 18 with the maximum amplitude of $\left|a_{11}\right|$ being 1.1. The corresponding results for $s_{22.23}^{1}$ were 7.5 and .52 , with the fractional value of equation (5.2) being 23 .

Using the result from the linear wave theory that the energy of a wave is proportional to the square of its amplitude we have the following conclusion. The extra modes (compared with $s_{22,23}^{1}$ ) did not increase the rate of change of $\left|a_{11}\right|$ but they enabled four times the amount of energy to be transferred to $a_{11}$ with a corresponding decrease in the average energy of $a_{2,22}$ and $a_{2,23}$. This increase in interface energy and decrease in surface energy compared with the three wave mode solution was found to occur in all our solutions with the effect, in general, being greater, the smaller $\mu$ was.

The next result to be taken from Figures 1 (a) and 1(b) is that the wave system, the centre of which is conveniently defined by the trough of the interface

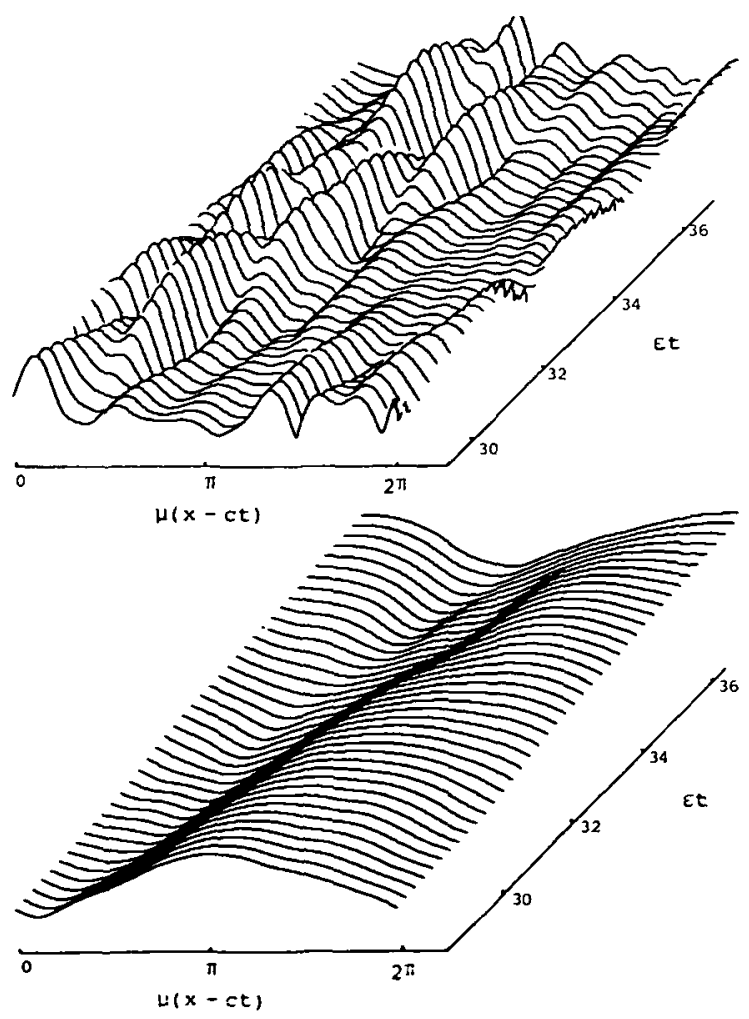

Figure 1(b). One wavelength of the surface envelope (upper graph) and interface displacement for $\tau=30-39$ with $\rho=1.05, \varepsilon=0.015, \mu .319$ and $K=22$. The vertical magnification is 25 . 
displacement, moves at a near constant velocity $\Delta C$ relative to the frame of reference moving with speed $d \omega_{A K} / d k \mu$. An estimate of $\Delta C$ can be made in the following way.

Assuming that the properties of the interface are dominated by the lowest interface mode $\left(a_{11}\right)$ and that locally in time about $t=t_{0}$ the magnitude of $a_{11}$ does not change, $a_{11}(t)$ can be written as

$$
a_{11}(t)=a_{11}\left(t_{0}\right) e^{-i \Delta c\left(t-t_{0}\right) \mu}
$$

for a small interval in time about $t=t_{0}$. Substituting into the evolution equations (3.2a) for this form of $a_{11}$ gives

$$
\Delta C=\left\{-\varepsilon \sum R_{A 1, l} a_{1 l}^{*} a_{1 /+1}-\varepsilon \sum R_{B 1, l} a_{2 l}^{*} a_{2 l+1}\right\} \frac{1}{\mu a_{11}} .
$$

The time average of this expression was found to obtain $\overline{\Delta C}$, which for this example was 0.009 while the value of $\overline{\Delta C}$ from Figures 1(a), 1(b) was 0.010 .

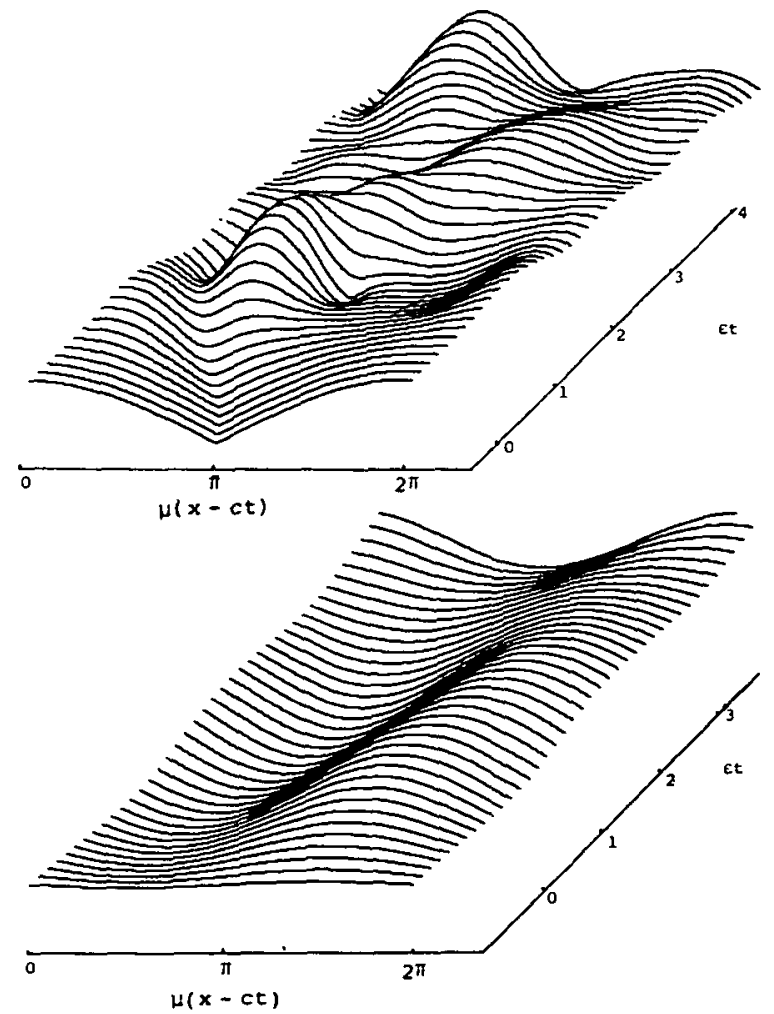

Figure 2(a). One wavelength of the surface envelope (upper graph) and interface displacement for $\tau=0-6$ with $\rho=1.05, \varepsilon=.006, \mu=1.35$ and $K=11$. The verticial magnification is 25 . 
The final result taken from the figures was that peaks in the envelope occurred above the region of convergence of the interface displacement and that the troughs of the interface displacement were narrower than the crests.

The second example considered was with $\rho=1.05, \varepsilon=.006, A=1.0, K=11$ and $\mu=1.35$.

Two interface modes and ten surface modes with wavenumbers seven to sixteen were required to describe the motion. This reduction in the number of modes compared with the first example follows from the larger value of $\mu$ giving a weaker interaction between modes on the two layers. The surface envelope and interface displacement are given in Figures 2(a) and 2(b) for two time intervals.

Referring to the figures the wave evolution as before was oscillatory and not strictly periodic. The graphs have a simpler structure than in the first example, a property that follows from the smaller number of modes present. The period $\left|a_{11}\right|$ was 4.6 with a maximum amplitude of 1.35 and the fractional value of equation (5.2) was 0.60 . For $s_{11,12}^{1}$ the period of $\left|a_{11}\right|$ was 3.5 and maximum amplitude 0.86 . Therefore as in the first example we have an increase in the amount of energy

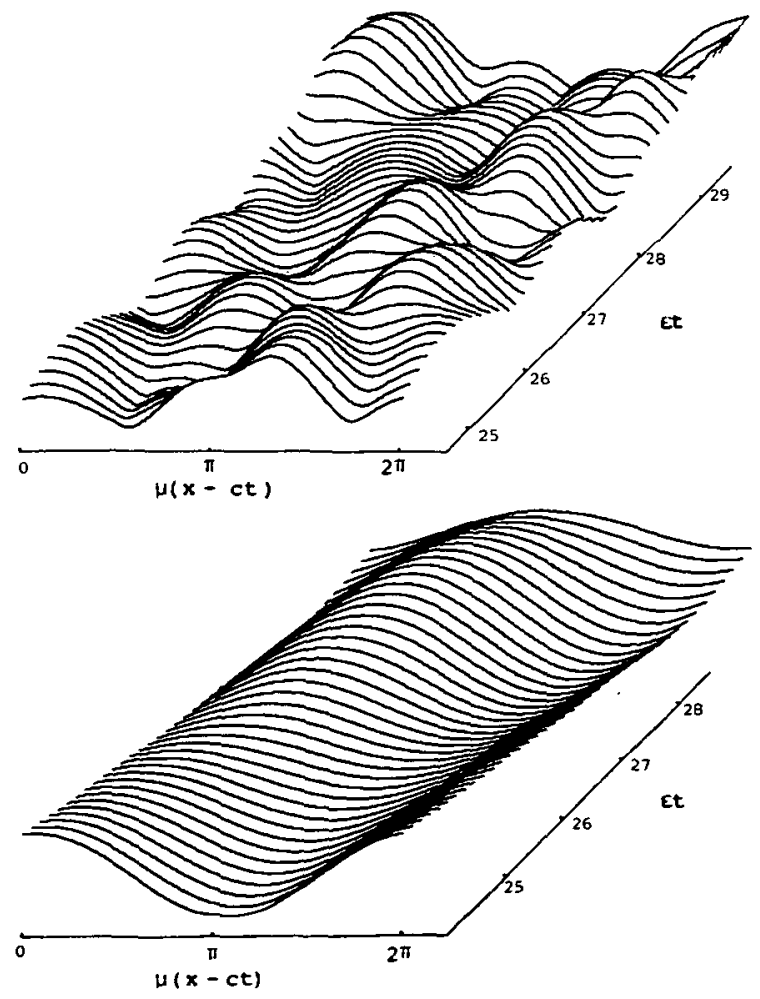

Figure 2(b). One wavelength of the surface envelope (upper graph) and interface displacement for $\tau=25-31$ with $\rho=1.05, \varepsilon=.006, \mu=1.35$ and $K=11$. The verticial magnification is 25 . 
transferred to $a_{11}$ compared with the three wavemode solution. However because the value of $\mu$ was larger the interaction between waves on the two layers was weaker giving a smaller increase in energy.

Using the method given in the first example, $\overline{\Delta C}$ was found to be 0.0019 which agrees to the accuracy quoted with that obtained from Figures 2(a) and 2(b). This improved agreement compared with the first example follows from the fact that there are only two interface modes with the time average amplitude of $a_{12}$ being 0.02 . Thus the assumption that the lowest interface mode dominates the interface is very realistic in this example.

\section{Discussion}

The method used here of having both the interface and surface displacements as unknown functions and allowing an arbitrary number of wave modes has enabled us to solve the unsteady wave problem (for small amplitude waves) on an open two layer fluid in greater generality than in the past.

We have found that although the analytical solutions with three wave modes present are periodic, the solutions when an arbitrary number of wave modes are present, are far from being periodic. We have also found no evidence that solutions tend asymptotically towards a strictly periodic solution. The addition of wave modes to complete the description of the motion allowed considerably more energy to be transferred to the interface and increased the period of evolution compared with the three wave mode solution. It was found in general that these effects increased as did the number of wave modes as $\mu$ decreased.

The results on the periodicity contrast with the work of Bryant [4] for the one layer case. Bryant found that the analytical solutions with three wave modes present were periodic, and increasing the number of wave modes made the solutions only slightly nonperiodic.

If the lower layer was assumed to be of finite and not infinite depth the analysis would be longer but we would expect similar results to those found here. However if $\mu$ was small, additional resonances would be possible and the evolution more complicated.

Finally, to illustrate the time scale and the strength of the nonlinear interactions considered in this paper the results from the first example in Section 6 are expressed as dimensioned quantities. Initially, two surface waves with wavelengths $45 \mathrm{~m}$ and $47 \mathrm{~m}$ respectively and each of $0.6 \mathrm{~m}$ amplitude generate an interface wave with wavelength $905 \mathrm{~m}$ and amplitude $0.93 \mathrm{~m}$. This takes 1380 seconds corresponding to 250 surface wave periods or 6 interface wave periods. 


\section{Acknowledgment}

The author wishes to thank Dr. P. J. Bryant for the discussion and encouragement given while writing this paper, the material in which formed part of the author's Ph.D. thesis.

\section{Appendix}

Defining

$$
\begin{aligned}
F_{k} & =\omega_{k}^{2} \cosh k \mu-k \mu \sinh k \mu, \\
G_{k} & =\omega_{k}^{2} \sinh k \mu-k \mu \cosh k \mu
\end{aligned}
$$

gives

$$
\begin{aligned}
R_{D k, l}= & \frac{\mu^{2} k^{2} T_{k}}{2 \omega_{k}^{2}\left(\rho+T_{k}\right)} \\
& \times\left[(\rho-1)\left(\omega_{l}^{2}-\omega_{l} \omega_{k+l}+\omega_{k+l}^{2}\right)\right. \\
& \quad-\rho\left(\omega_{k+l}^{2}-\omega_{l}^{2}\right)-\rho \omega_{l} \omega_{k+l}+\omega_{l} \omega_{k+l} \frac{G_{l} G_{k+l}}{F_{l} F_{k+l}} \\
& +\left(\omega_{k+l}-\omega_{l}\right)\left(\frac{\rho}{T_{k}}-\frac{(\rho-1) \mu k}{\omega_{k}^{2}}\right)\left(\omega_{k+l} \frac{G_{k+l}}{F_{k+l}}+\omega_{l} \frac{G_{l}}{F_{l}}\right) \\
& \left.+\frac{\mu k F_{k}\left(\omega_{k+l}-\omega_{l}\right)\left(\frac{(k+l) \mu}{\omega_{k+l}^{2}}+\frac{l \mu}{\omega_{l}}\right) \frac{\omega_{l}^{2} \omega_{k+l}^{2}}{F_{l} F_{k+l}},}{\omega_{k}^{2} F_{l} F_{k+l}}\left(\omega_{k+l}^{2} F_{k}-\omega_{l} \omega_{k+l}+\omega_{k+l}^{2}-\mu l \frac{(k+l) \mu}{\omega_{l} \omega_{k+l}}\right)\right] \\
R_{E k, l}=\frac{1}{2} F_{k}\left[(\rho-1)\left(\omega_{l}^{2}-\omega_{l} \omega_{k+l}+\omega_{k+l}^{2}\right)-\rho\left(\omega_{k+l}^{2}-\omega_{l}^{2}\right)\right. & \left.-\left(\omega_{k+l}-\omega_{l}\right) \mu k\left(\frac{(k+l)}{\omega_{k+l}}+\frac{l \mu}{\omega_{l}}\right)\right] \\
& +\frac{1}{2} G_{k}\left(\omega_{k+l}-\omega_{l}\right)\left(\omega_{k+l} \frac{G_{k+l}}{F_{k+l}}+\omega_{l} \frac{G_{l}}{F_{l}}\right) \\
& +\frac{1}{2} \frac{\omega_{l}^{2} \omega_{k+l}^{2}}{F_{l} F_{k+l}}\left[\omega_{k+l}^{2}\left(\omega_{l}^{2}-\omega_{l} \omega_{k+l}+\omega_{k+l}^{2}-\frac{\mu_{l}^{2} l(k+l)}{\omega_{l} \omega_{k+l}}\right)\right.
\end{aligned}
$$




$$
\begin{aligned}
& R_{A k, l}=\left[\frac{\omega_{k}^{4}\left(\omega_{k+l}-\omega_{l}-\omega_{k}\right) R_{D k, l}}{\left(\omega_{k+l}-\omega_{l}\right)^{2}-\left(\omega_{A k} \omega_{B k} / \omega_{k}\right)^{2}}+\frac{\omega_{k}^{2} F_{k} R_{E k, l}}{\omega_{k+l}-\omega_{l}+\omega_{k}}\right] /\left((\rho-1) F_{k}^{2}+\omega_{k}^{4}\right) \\
& \text { with } \omega_{k}=\omega_{B k}, \omega_{l}=\omega_{B l}, \omega_{k+l}=\omega_{B k+l} . \\
& R_{B k, l}=\frac{F_{l} F_{k+l}}{\omega_{l}^{2} \omega_{k+l}^{2}}\left[\frac{\omega_{k}^{4}\left(\omega_{k+l}-\omega_{l}-\omega_{k}\right) R_{D k, l}}{\left(\omega_{k+l}-\omega_{l}\right)^{2}-\left(\omega_{A k} \omega_{B k} / \omega_{k}\right)^{2}}\right. \\
& \left.+\frac{\omega_{k}^{2} F_{k} R_{E k, l}}{\omega_{k+l}-\omega_{l}+\omega_{k}}\right] /\left((\rho-1) F_{k}^{2}+\omega_{k}^{2}\right) \\
& \text { with } \omega_{k}=\omega_{B k}, \omega_{l}=\omega_{A l}, \omega_{k+l}=\omega_{A k+l} \text {. } \\
& R_{C k, l}=\left[\frac{\omega_{k}^{4} R_{E k, l}}{\omega_{k+l}-\omega_{l}+\omega_{k}}\right. \\
& -\frac{(\rho-1) F_{k} \omega_{k}^{2}\left(\omega_{k+1}-\omega_{l}-\omega_{k}\right) R_{D k, l}}{\omega_{k+1}-\omega_{l}+\omega_{k}} \\
& \left.-\frac{(\rho-1) F_{k} \omega_{k}^{2}\left(\omega_{k+l}-\omega_{l}-\omega_{k}\right) R_{D k, l}}{\left(\omega_{k+l}-\omega_{l}\right)^{2}-\left(\omega_{A k} \omega_{B k} / \omega_{k}\right)^{2}}\right] \frac{F_{k+l}}{\omega_{k+l}^{2}} /\left((\rho-1) F_{k}^{2}+\omega_{k}^{4}\right) \\
& \text { with } \omega_{k}=\omega_{A k}, \omega_{l}=\omega_{B l}, \omega_{k+l}=\omega_{A k+l} \text {. }
\end{aligned}
$$

Although the expressions for $R_{D k, l}$ and $R_{E k, l}$ simplify when the explicit forms of the frequencies $\omega_{k}, \omega_{l}$ and $\omega_{k+l}$ are substituted the above form was retained to increase the efficiency of the program coding for the computer.

\section{References}

[1] F. K. Ball, "Energy transfer between external and internal gravity waves", J. Fluid Mech. 19 (1964), 465-478.

[2] D. J. Benney, "Significant interaction between small and large scale surface waves", Stud. Appl. Math. 55 (1976), 93-106.

[3] F. P. Bretherton, "Resonant interactions between waves. The case of discrete oscillations", $J$. Fluid Mech. 20 (1964), 457-479.

[4] P. J. Bryant, "Periodic waves in shallow water", J. Fluid Mech. 59 (1973), 625-644.

[5] P. J. Bryant, "Permanent wave structures on an open two layer fluid", Stud. Appl. Math. 59 (1977), 225-246.

[6] Yasuo Hasizume and Norito Ikeda, "Resonant interaction of waves on a stratified fluid", $J$. Phys. Soc. Japan 45 (1978), 665-673.

[7] H. Lamb, Hydrodynamics, 3rd ed. (Cambridge University Press, 1906), 355-356. 
[8] O. M. Phillips, "On the dynamics of unsteady gravity waves of finite amplitude: Part 1: The elementary interactions", J. Fluid Mech. 9 (1960), 193-217.

[9] O. M. Phillips, The dynamics of the upper ocean, 2nd ed. (Cambridge University Press, 1977), 38.

[10] M. H. Rizk and D. R. S. Ko, "Interaction between small scale surface waves and large scale internal waves", Phys. Fluids 21 (1978), 1900-1907.

[11] L. F. Shampine and M. K. Gordon, Computer solution of ordinary differential equations. The initial value problem (W. H. Freeman and Co., 1975).

[12] P. W. Sharp, Ph.D. Thesis, University of Canterbury, 1983.

[13] K. M. Watson, B. J. West and B. I. Cohen, "Coupling of surface and internal gravity waves: a mode coupling model”, J. Fluid Mech. 77 (1976), 185-208. 